\title{
The Social Hierarchy of Female Macaca Fascicularis Increased by Successful Reproduction
}

\author{
Zhilong Wang ${ }^{1}$, Jiayuan Peng ${ }^{2}$, Weizhong Chen ${ }^{3}$, Fangfang Liu ${ }^{4}$, Wei Ren ${ }^{5}$ \\ Jun Xie6, Chenying Yan ${ }^{6}$, Jing Zhong ${ }^{6}$, Greg Mirt ${ }^{7}$, Peter Pohl ${ }^{8}$, Fan $\mathrm{Xu}^{3^{*}}$ \\ ${ }^{1}$ The Engineering \& Technical College of Chengdu University of Technology, Sichuan, China \\ ${ }^{2}$ Department of Basic Medical Science, Chengdu Medical College, Sichuan, China \\ ${ }^{3}$ Department of Public Health, Chengdu Medical College, Sichuan, China \\ ${ }^{4}$ Southwest Minzu University, Sichuan, China \\ ${ }^{5}$ Department Of Humanities, Chengdu Medical College, Sichuan, China \\ ${ }^{6}$ Department Of Pharmacy, Chengdu Medical College, Sichuan, China \\ ${ }^{7}$ Occupational Activity Centre Novo Mesto, Slovenia, EU \\ ${ }^{8}$ Child Psychology Practice Garmisch, Garmisch-Partenkirchen, Germany
}

*Corresponding Author: Prof XU Fan, Public Health School, Chengdu Medical College, Xindu Avenue, Xindu District, Sichuan Province, China

\begin{abstract}
The Social hierarchy naturally occurs within numerous species, which maintain the social structure, relationship with the ecosystem and sustainable reproduction. However, the formation of social hierarchy in primate society remains unclear. Here we have used our previous published open data, monkeys' daily behaviors, using the Structural Equation Model (S.E.M.) to construct the response variables, which affect the subject's social rank. These results revealed that the social hierarchy of female subjects may increase due to their reproduction contribution to the society. This kind of S.E.M. analysis may help us discover if the inner structure, really exists but yet unknown, in psychological and sociological sociology researches.
\end{abstract}

Keywords: Primate Society, Social structure, Social hierarchy, Structural Equation Model.

\section{INTRODUCTION}

The social hierarchy exists as one social organization that established by fighting or display behaviour and results in a ranking of the animals in a group [1]. Due to its selective advantage in an extensive range of ecology, it exists within numerous species including insects, crustaceans, and mammals, monkeys, raven and human. The social hierarchy maintains the relative stability of the community structure and sustainable reproduction in species. In most social species, the dominance rank can reduce the community tension and frequency of mutual attacks, namely enhance the friendship in a group [2]. Moreover, it can be affect the reproductive rate and the health of animals [3].

Crucially, the social rank exists extensively in ecology. For example, dominant insects can benefit more including the reproduction and food source during the lifespan according to dominant social rank than subordinate[4, 5]. While in ant society, many individuals live and work together and perform different roles in their lifetime. The queen lays eggs, the worker ants cover most jobs, including collecting food, caring for the young and protecting the colony [6]. Furthermore, social grade sites are also widely found in fish [7], social structures [8, 9] and spatial memory and manipulation of the environment [10].

An intelligent bird, ravens have a complex social structure as well. Male's social rank is higher than female. Common ravens are renowned for their sophisticated socio-cognitive skills such as tactical deception [11], gaze following, perspective taking [12,13], and possibly even knowledge attribution [13]. 
Moreover, many non-human primate species construct a linear hierarchy in their societies $[14,15]$ according to their dominance rank and linkage [16].

The formation of social hierarchies is a natural occurring and evolutionarily conserved phenomenon, with profound influences on health and sickness [17, 18], stabilized social networks [19], maintain and regulate the social welfare and health of individuals within a group[20]. Carrie et al's study revealed that social hierarchy affects mental health in part through a process of emotional suppression [21].

Our previous works have constructed the comprehensive and systemic ethogram of Macaca fascicularis in free enclosure [22]. While an interesting phenomenon was found that female monkeys' behaviors would be affected by their infants [23], and they were resting much more time in the dominant place with the high social rank male subjects than who were not reared the infant [24]. However, the causal inference or latent relationship remains unclear. Therefore, we hypothesized that the social hierarchy of female subject present higher than who are not reared the infant Furthermore, to validate this hypothesis, we used the Structural Equation Model (S.E.M.) to disclose social hierarchy influencing factors of Macaca fasciculari. This kind of analysis may provide a comprehensive perspective on the impact of social hierarchy on cognitive behavior, psychological stress and physical disease.

\section{METHOD}

\subsection{Data Sources}

Here we used our previous studies' data, systematic ethogram of Macaca fascicularis in a free enclosure [22], the mutual influence between Macaca fascicularis mothers and their infants [23], and naturally occurring depression on monkey [24]. All of these filed observations provided the details of daily behaviours of Macaca fascicularis in the free enclosure and all data are open for public for further investigations [22-24].

\subsection{Variables Selection Process}

According to the previous work, the higher-ranking monkeys were staying more time on the board (a relative comfortable resting place in enclosure) when they were foraging or resting, which reflect the monkey's territorial consciousness. [22]. In addition, the higher-ranking monkeys showed high frequency drinking behaviour and grooming or being groomed; while lower-ranking monkeys spent more time staying at the edge of the cage or climbing on other objects. It is obvious that they were often crowded together. Similar to Shively's study [25], monkeys of the subordinates were found to be relatively inactive and they can get warm by keeping close to other bodies. Therefore, the following variables/behaviours, including Walking on the board (LWD), Mutual grooming (AMG), Drinking (IDR), Feeding while sitting on the board (IFA), Climbing rest (RCR), and Embrace (TEM), were used to perform social hierarchy scale on monkeys.

\subsection{Data Cleaning}

The daily observation time is divided into 8 phases, 4 in the morning and 4 in the afternoon, 30 minutes per phase. The data included 40 monkeys in 8 different days, we selected the data from the same monkey in the same day. The behaviour of the phase is summed as an independent observation. Total duration of each behaviour among 8-time phases was used to perform the analysis and delete the data if one subject pseudo replicated.

\subsection{Data Normality Test}

Data normality test was performed firstly to access the accuracy of model. The monkey behaviours, reared in the free enclosure, presented asymmetrically via skewness and kurtosis analysis, more details please see table 1. Furthermore, CFI values larger than 0.90 and RMSEA less than 0.10, these indicated that this model fit for current situation.

Table1. Behaviour Data Distribution

\begin{tabular}{|c|c|c|c|c|c|c|}
\hline Actions & $\mathrm{N}$ & Mean & Std. dev. & Median & Interquartile Range & P-value $^{\wedge}$ \\
\hline LWD $^{*}$ & 168 & 34.36 & 58.54 & 4.345 & 48.57 & $<0.0001$ \\
\hline AMG* $^{*}$ & 168 & 531.08 & 636.91 & 263.225 & 824.82 & $<0.0001$ \\
\hline IDR $^{*}$ & 168 & 35.64 & 61.42 & 1.845 & 44.315 & $<0.0001$ \\
\hline IFA $^{*}$ & 168 & 37.28 & 94.32 & 0 & 0 & $<0.0001$ \\
\hline
\end{tabular}


The Social Hierarchy of Female Macaca Fascicularis Increased by Successful Reproduction

\begin{tabular}{|c|c|c|c|c|c|c|}
\hline RCR* & 168 & 1188.91 & 2160.53 & 156.93 & 1321.6 & $<0.0001$ \\
\hline TEM* $*$ & 168 & 2488.36 & 2488.97 & 1806.35 & 3637.4 & 0.0001 \\
\hline \multicolumn{7}{|c|}{$\begin{array}{l}\text { *LWD= Walking on the board; } \mathrm{AMG}=\text { Mutual grooming; IDR= Drinking; IFA= Feeding while sitting on the } \\
\text { board; } \mathrm{RCR}=\text { Climbing rest; } \mathrm{TEM}=\text { Embrace } \\
\text { Shapiro-Francia W' test }\end{array}$} \\
\hline
\end{tabular}

\subsection{Statistical Analysis}

Spearman correlation analysis wasused between all variables to classify their relationships firstly. Then, SEM procedure with the Maximum Likelihood Estimation was preformed. Generally, the SEM consists of measurement and structural models. Measurement model indicates latent variables with observed variables, and structural model reflects the causal relationship between latent variables due to our previous hypothesis. Correlation coefficients between behaviours revealed the interrelationship of them. Next, we constructed a category affect model of social hierarchy of Macaca fascicularis. Finally, S.E.M. analysis was performed to calculate the standardization coefficient and correlation coefficient for each variable. All analyses procedure was performed using STATA12.0 software.

\section{Results}

\subsection{Correlation Analysis}

To validate the latent relationship of this phenomenon, we performedspearman correlation analysis among all variables. Interestingly, the correlation coefficient between first four behaviours (LWD, AMG, IDR, IFA against LWD AMG IDR and IDR) were positive, however the correlation coefficient of first four behaviours between the last two behaviours (LWD, AMG, IDR, IFA against RCR, TEM) were negative, for details please see table 2 .

Table2. The Correlation matrix between behaviours

\begin{tabular}{|c|c|c|c|c|c|c|}
\hline Actions & LWD & AMG & IDR & IFA & RCR & TEM \\
\hline LWD & 1 & & & & & \\
\hline AMG & 0.342 & 1 & & & & \\
\hline IDR & 0.270 & 0.309 & 1 & & & \\
\hline IFA & 0.125 & 0.166 & 0.149 & 1 & & 1 \\
\hline RCR & -0.272 & -0.231 & -0.082 & -0.077 & 1 & \\
\hline TEM & -0.218 & -0.270 & -0.236 & -0.132 & -0.052 & 1 \\
\hline \multicolumn{7}{|c|}{ Footnote: The data in the table is symmetrical } \\
\hline
\end{tabular}

\subsection{Structural Equation Model (S.E.M)}

In S.E.M model, we defined the "reared baby" as the exogenous variable, namely the female monkey hold its infant; meanwhile "Social Hierarchy" defined as the endogenous latent variable, it can be redirect reflected by relationship with king monkey in this colony. Namely, there is only one factor (reared baby or not) affects the social hierarchy of female monkeys. The path analysis in S.E.M. disclosed that the coefficient of impact of baby on Social Hierarchy is 0.52 . This signifiedreared the baby may contribute the social hierarchy of female monkey by $52 \%$. The scheme of S.E.M., please see Figure 1.

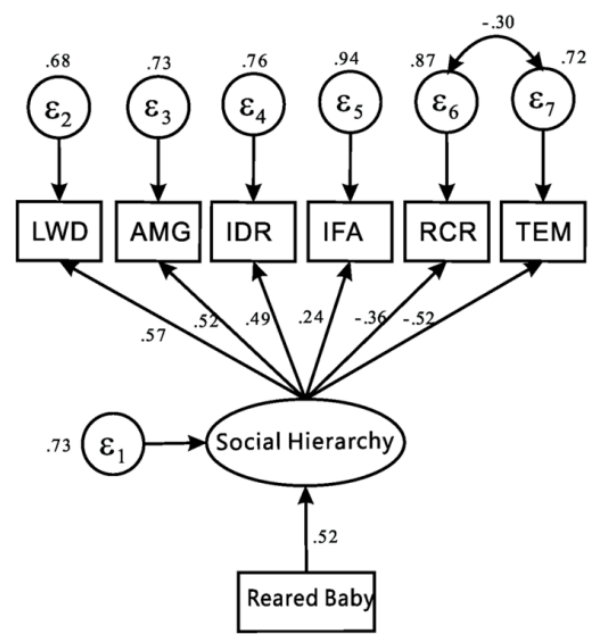

Fig1.The Scheme of S.E.M of Social Hierarchy of Female Monkey 
Footnote: Variable "Reared Baby" has a value of 0 or 1 , when taken 0 means the monkey does not reared a baby, and 1 indicates that the monkey reared a baby. Circles indicate the latent variables and Rectangles represent observed variables. Significant paths with their estimated parameter are shown lines. Standardized path coefficients are presented at the midpoint of the unidirectional arrow paths.

According to the positive coefficient of behaviours including LWD AMG IDR and IFA, it can be inferred that these behaviours were positively related to social hierarchy. On the contrary, the negative coefficient indicated that the behaviours (RCR and TEM) were negatively related to social hierarchy. The larger was the absolute value of the coefficient of behaviour to social hierarchy, the greater impact it has on social hierarchy. For example, the coefficient of LWD to social hierarchy is 0.57 (please see Fig 1), which is greater than the coefficient of AMG (0.52) to social hierarchy. This signified that the social hierarchy has larger impact than on LWD than AMG.

Table3. Structural Equation Model of Social Hierarchy of Female Monkey

\begin{tabular}{|c|c|c|c|c|}
\hline S.E.M Model & Path/measurement & Coef. (unstandard) & Coef. (Standard) & $\boldsymbol{P}$ \\
\hline Structural & $\begin{array}{c}\text { Reared Baby -> Social } \\
\text { Hierarchy }\end{array}$ & 37.75 & 0.52 & $<0.0001$ \\
\hline \multirow[t]{6}{*}{ Measurement } & Social Hierarchy -> LWD & 1(constrained) & 0.57 & $<0.0001$ \\
\hline & Social Hierarchy -> AMG & 9.97 & 0.52 & $<0.0001$ \\
\hline & Social Hierarchy -> IDR & .90 & 0.49 & $<0.0001$ \\
\hline & Social Hierarchy -> IFA & .69 & 0.24 & 0.006 \\
\hline & Social Hierarchy $->$ RCR & -23.37 & -0.36 & 0.001 \\
\hline & Social Hierarchy $->$ TEM & -39.37 & -0.52 & $<0.0001$ \\
\hline \multirow[t]{7}{*}{ Variance } & e.LWD & 2311.98 & 0.68 & \\
\hline & e.AMG & 294452.4 & 0.73 & \\
\hline & e.IDR & 2854.04 & 0.76 & \\
\hline & e.IFA & 8328.40 & 0.94 & \\
\hline & e.RCR & 4042375 & 0.87 & \\
\hline & e.TEM & 4460856 & 0.72 & \\
\hline & e.SocialHirerarchy & 796.91 & 0.73 & \\
\hline Covariance & e.RCR $*$ e.TEM & -1283876 & -0.30 & 0.001 \\
\hline \multicolumn{5}{|c|}{$*$ Goodness of fit: Chi-square $(13)=23.85, P=0.0326, C F I=0.911, R M S E A=0.070, S R M R=0.046$} \\
\hline
\end{tabular}

The coefficient of Social Hierarchy to the first four behaviours including LWD, AMG, IDR and IFA, were positive, this signified that social hierarchy has positive effect on corresponding behaviours. Namely, if social hierarchy was increased, and its corresponding behavior may increase as well to some extent, more details listed in table 3. For instance, the impact of Social Hierarchy on LWD is 0.57 . On the other hand, if social hierarchy increased by one unit, then the corresponding behaviour LWD may increase by $57 \%$.

Moreover, the coefficient of Social Hierarchy to the last two behaviors, RCR and TEM, were negative, which indicated that social hierarchy has the negative effect on corresponding behaviours, please see Fig 1 and Table 3. More particularly, if social hierarchy were increased, the duration of its corresponding behaviour may decrease. For example, the coefficient of Social hierarchy on RCR is 0.36 , that means if social hierarchy increased by one unit, and the corresponding behaviour LWD reduce by $36 \%$.

\section{DISCUSSION}

By means of S.E.M., we have got clear relationship of reared infant female monkey social hierarchy modified in the social colony. The female monkey presented more time in activities such as "Walking on the board", "Mutual grooming", "Drinking", "feeding while sitting on the board" and less time in activities such as climbing rest and embracing.

The significant different behaviours can be classified into ingestion, social and territory-related behaviour categories according to the ecology function. With regarding to the ingestion behaviour, we found that monkeys with higher social rank spent more time on "walking on the board" and "feeding while sitting on the board". These behaviours were similar with the results of Erik P. Willems' study, which disclosed that primates have a sense of maintaining collective interests and territory, more particular in their individual activity space. [26]. While Post DG found out that in the society of 
yellow baboons, high-ranking individuals generally had longer feeding bout durations than lowerranking individuals [27].

Another interesting category is their social behaviour, mutual grooming [28, 29] and embrace with company. Our data revealed that monkeys with higher social ranking are good at interacting with other members and spent more time on mutual grooming. These features were consistent with Albert SC' study, they found that collective action and/or the exchange of social resources are prominent features of baboon social life. Adult females with higher social dominance rank have accelerated reproduction and engage in more frequent social interactions such as grooming [30]. Wu CFet al found that grooming between non-kin individuals will be directed up the dominance rank of Macaca mulatta brevicaudus[31], please refer the Figure 2.

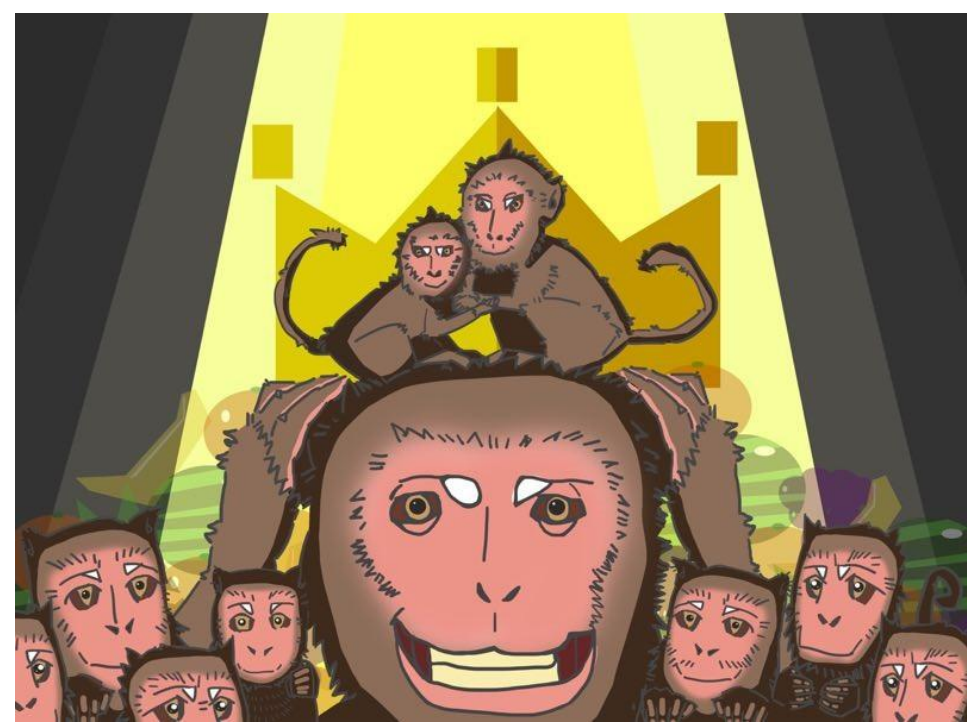

Fig2. Social hierarchy in social groups of monkey

With respecting to embrace behaviour, we found that lower social rank monkeys much more preferred to be embraced with companies. Similar to Shively's study [25], the subordinate monkeys were found to be relatively inactive and they can get warm by keeping close to other bodies. Moreover, among subordinate monkeys were less responsive to environmental stimuli, and the vigilance of another monkey.

Another important category is territory-related behaviour, monkeys with lower social rank spent more time on hanging their bodies to rest to facilitate observation of their companions, were in a state of higher vigilance. This is similar to the findings of Evers E [32], the social vigilance behaviour of individuals with different social ranks has significant individual differences among the primate social groups.

Due to the S.E.M. procedure, every variable has been checked, this may help us consider these latent variables and their inner relationship comprehensively. Furthermore, the measurement error of the independent variable and dependent variable are acceptable. Also, each latent variable can be measured through other causal inference. Factor structure and the factor relationship were running simultaneously.

\section{CONCLUSION}

In conclusion, the social hierarchy of female monkey may increase due to reared the infant who not, and they spent more time on "walking on the board", "mutual grooming", "sitting on the board", less time on "climbing rest on the window" and "embracing with company". These behaviours could be reflecting their social rank indirectly via structural equation modeling. This study may open new perspectives for the neurosciences of social dominance.

\section{Limitation}

Generally, the value of RMSEA need to be lower than 0.05 according to the principle of S.E.M. However, it is also acceptable for asymmetric distribution data. Its value is 0.07 , lower than 0.10 , this may be shortened the reliability, therefore, more sample sizes or observations are needed furthermore. 


\section{ACKNOWLEDGEMENT}

This research was supported by the Population and health project of Chengdu Municipal Science and Technology Bureau (10YTYB272SF-182), the Science \& Technology Department of Sichuan Province Grants (2016JY0036), Chengdu, China.

\section{REFERENCES}

[1] Fiske, S.T., Interpersonal Stratification: Status, Power, and Subordination. 2010: p.941-982.

[2] Ellis, L., Dominance and reproductive success among nonhuman animals: A cross-species comparison. Ethol Sociobiol, 1995. 16(4): p. 257-333.

[3] Sapolsky, R.M., The influence of social hierarchy on primate health. Science, 2005. 308(5722): p. 648-52.

[4] Ratnieks, F.L., K.R. Foster and T. Wenseleers, Conflict resolution in insect societies. Annu Rev Entomol, 2006. 51: p. 581-608.

[5] Lukas, D. and T. Clutton-Brock, Social complexity and kinship in animal societies. Ecol Lett, 2018. 21(8): p. 1129-1134.

[6] Mikheyev, A.S. and T.A. Linksvayer, Genes associated with ant social behavior show distinct transcriptional and evolutionary patterns. Elife, 2015. 4: p. e04775.

[7] Brown, C., K. Laland and J. Krause, Fish cognition and behavior (series: fish and aquatic resources). Fish Cognition \& Behavior, 2011. 67(Dec): p. F2D-1 - F2D-3.

[8] Timms, A.M. and M.H. Keenleyside, The reproductive behavior of Aequidens paraguayensis (Pisces, Cichlidae). Z Tierpsychol, 1975(39): p. 8-23.

[9] Pasko, L., Tool-like behavior in the sixbar wrasse, Thalassoma hardwicke (Bennett, 1830). Zoo Biol, 2010. 29(6): p. 767-73.

[10] Hughes, R.N. and C.M. Blight, Algorithmic behaviour and spatial memory are used by two intertidal fish species to solve the radial maze. Anim Behav, 1999. 58(3): p. 601-613.

[11] Bugnyar, T. and K. Kotrschal, Observational learning and the raiding of food caches in ravens, Corvus corax : is it 'tactical' deception? Animal Behaviour, 2002. 64(2): p. 185-195.

[12] Bugnyar, T., M. Stowe and B. Heinrich, Ravens, Corvus corax, follow gaze direction of humans around obstacles. Proc Biol Sci, 2004. 271(1546): p. 1331-6.

[13] Bugnyar, T. and B. Heinrich, Ravens, Corvus corax, differentiate between knowledgeable and ignorant competitors. Proc Biol Sci, 2005. 272(1573): p. 1641-6.

[14] de Waal, F.B., Macaque social culture: development and perpetuation of affiliative networks. J Comp Psychol, 1996. 110(2): p. 147-54.

[15] Cheney, D.L. and R.M. Seyfarth, How monkeys see the world: Inside the mind of another species. 1995. 482-483.

[16] Bergman, T.J., et al., Hierarchical classification by rank and kinship in baboons. Science, 2003. 302(5648): p. 1234-6.

[17] Zink, C.F., et al., Know your place: neural processing of social hierarchy in humans. Neuron, 2008. 58(2): p. 273-83.

[18] Nagy, M., et al., Hierarchical group dynamics in pigeon flocks. Nature, 2010. 464(7290): p. 890-3.

[19] Ridgeway, C.L., Status Construction Theory. 2015: John Wiley \& Sons, Ltd.

[20] Boyce, W.T., Social stratification, health, and violence in the very young. Ann N Y Acad Sci, 2004. 1036: p. 47-68.

[21] Langner, C.A., et al., Social hierarchy and depression: the role of emotion suppression. J Psychol, 2012. 146(4): p. 417-36.

[22] $\mathrm{Xu}, \mathrm{F}$., et al., Construction and validation of a systematic ethogram of Macaca fascicularis in a free enclosure. PLoS One, 2012. 7(5): p. e37486.

[23] Zhou, Q., et al., The mutual influences between depressed Macaca fascicularis mothers and their infants. PLoS One, 2014. 9(3): p. e89931.

[24] Xu, F., et al., Macaques exhibit a naturally-occurring depression similar to humans. Sci Rep, 2015. 5: p. 9220.

[25] Shively, C.A., et al., Social stress-associated depression in adult female cynomolgus monkeys (Macaca fascicularis). Biol Psychol, 2005. 69(1): p. 67-84.

[26] Willems, E.P., B. Hellriegel and C.P. van Schaik, The collective action problem in primate territory economics. Proc Biol Sci, 2013. 280(1759): p. 20130081. 
[27] Post, D.G., G. Hausfater and S.A. McCuskey, Feeding behavior of yellow baboons (Papio cynocephalus): relationship to age, gender and dominance rank. Folia Primatol (Basel), 1980. 34(3-4): p. 170-95.

[28] Kummer, H., The Social Organization of Hamadryas Baboons: A Field Study. Quarterly Review of Biology, 1968. 19(4).

[29] Rowell, T., The social behaviour of monkeys. 1972: Penguin Books.

[30] Alberts, S.C., Social influences on survival and reproduction: Insights from a long-term study of wild baboons. J Anim Ecol, 2018.

[31] Wu, C.F., et al., The influence of kinship and dominance hierarchy on grooming partner choice in freeranging Macaca mulatta brevicaudus. Primates, 2018. 59(4): p. 377-384.

[32] Evers, E., et al., Look before you leap - individual variation in social vigilance shapes socio-spatial group properties in an agent-based model. Behav Ecol Sociobiol, 2012. 66(6): p. 931-945.

Citation: Fan Xu, et.al. "The Social Hierarchy of Female Macaca Fascicularis Increased by Successful Reproduction”. International Journal of Research Studies in Biosciences (IJRSB). 7(12), pp. 1-7. DOI: http://dx.doi.org/10.20431/2349-4050.0712001

Copyright: () 2019 Authors this is an open-access article distributed under the terms of the Creative Commons Attribution License, which permits unrestricted use, distribution, and reproduction in any medium, provided the original author and source are credited. 